\title{
On the Relationship between the Generalized Likelihood Ratio Test and Backprojection for Synthetic Aperture Radar Imaging
}

\author{
K. Voccola ${ }^{a}$, B. Yazıc1 ${ }^{a}$, M. Ferrara ${ }^{b}$, M. Cheney ${ }^{a}$ \\ ${ }^{a}$ Rensselaer Polytechnic Institute, 110 8th Street, Troy, NY, USA; \\ ${ }^{b}$ Air Force Research Lab, Dayton, OH, USA
}

\begin{abstract}
In synthetic aperture radar (SAR) imaging, ${ }^{1}$ a scene of interest is illuminated by electromagnetic waves. The aim is to reconstruct an image of the scene from the measurement of the scattered waves using airborne antenna(s). There are many imaging systems which are built upon this notion such as mono-static SAR, bi-static SAR, ${ }^{2}$ and hitchhiker SAR. ${ }^{3}$ For these modalities, there are analytic reconstruction algorithms based on backprojection. Backprojection-based algorithms have the advantage of putting the visible edges of the scene at the right location and orientation in the reconstructed images.

On the other hand, there is also a SAR imaging method based on the generalized likelihood-ratio test (GLRT). In particular we consider the problem of detecting a target at an unknown location. In the GLRT, the presence of a target in the scene is determined based on the likelihood-ratio test. Since the location of the target is not known, the GLRT test statistic is calculated for each position in the scene and the location corresponding to the maximum test statistic indicates the location of a potential target.

In this paper, we show that the backprojection-based analytic reconstruction methods include as a special case the GLRT method. We show that the GLRT test statistic is related to the reflectivity of the scene when a backprojection-based reconstruction algorithm is used.
\end{abstract}

Keywords: synthetic aperture radar, filtered backprojection, generalized likelihood ratio test

\section{INTRODUCTION}

There are at least two approaches to forming Synthetic-Aperture Radar (SAR) images: one based on filtered backprojection, and the other using the generalized likelihood ratio test from statistical detection-and-estimation theory. This paper establishes a connection between these two approaches. A similar connection was noted by Rossi and Willsky ${ }^{4}$ for image formation using the Radon transform in the case when the image is parametrized by a finite number of parameters.

In this paper, we first show how a number of SAR modalities can be put into a common framework. We then describe the image-formation approach based on filtered backprojection. Lastly we outline an image-formation technique which arises from a continuum version of the generalized likelihood ratio test. In particular, we obtain a spatially-varying test statistic, a plot of which is an image of the scene. We show that the formula for the test statistic has the form of filtered backprojection.

\section{SYNTHETIC APERTURE RADAR IMAGING}

In SAR, one forms an image of a region from measurements of electromagnetic waves made from one or more airborne antenna(s) flying above the region. We first describe the form the measurements take in SAR and then detail how an image is formed using an analytic filtered-backprojection reconstruction method.

Further author information: Send correspondence to K.Voccola

E-mail: voccok@rpi.edu

Automatic Target Recognition XIX, edited by Firooz A. Sadjadi, Abhijit Mahalanobis,

Proc. of SPIE Vol. 7335, 73350I - @ 2009 SPIE - CCC code: 0277-786X/09/\$18 - doi: 10.1117/12.818554

Proc. of SPIE Vol. 7335 73350l-1

Downloaded from SPIE Digital Library on 17 Feb 2010 to 128.113.62.199. Terms of Use: http://spiedl.org/terms 


\subsection{Model for SAR data}

The received data can be thought of as weighted integrals of a function $T$ over some curved manifolds such as circles, ellipses, or hyperbolas. The function $T$ models the reflectivity or irradiance of the region of interest. We consider three different SAR modalities: mono-static SAR, bi-static SAR, and hitchhiker SAR.

Throughout this paper we use the following font conventions: bold face italic font $(\boldsymbol{x})$ denotes two-dimensional vectors and bold face roman font $(\mathbf{x})$ denotes three-dimensional vectors. Also latin capital letters in calligraphic font $(\mathcal{F})$ are used for operators.

i.) Mono-static SAR: In this case we assume that the antenna travels along the path $\gamma(s) \in \mathbb{R}^{3}$. It is known ${ }^{1}$ that the received signal or data at a point $\gamma(s)$ and time $t$ can be modeled using the Born approximation (a.k.a. the single-scattering approximation) as follows:

$$
d(s, t)=\mathcal{F}[T](s, t)=\int e^{-i \omega\left(t-r_{s, \mathbf{x}}\right)} A(\boldsymbol{x}, s, \omega) T(\boldsymbol{x}) d \boldsymbol{x} d \omega,
$$

where

$$
r_{s, \mathbf{x}}=2|\gamma(s)-\mathbf{x}| / c_{0}
$$

and $\boldsymbol{x}=\left(x_{1}, x_{2}\right), \mathbf{x}=(\boldsymbol{x}, 0)$. For simplicity we assume flat topography. We refer to $t$ as the fast-time variable and $s$ as the slow-time variable. Also note $c_{0}$ denotes the speed of light and $\omega$ is the temporal frequency. $T$ is the function modeling the reflectivity of the region to be imaged and $A(\boldsymbol{x}, s, \omega)$ is an amplitude depending on geometrical spreading factors, antenna beam pattern, transmitted waveform, and antenna trajectories.

The method of stationary phase can be used to show that the main contributions to (1) come from the critical set

$$
X=\left\{\left(x_{1}, x_{2}, 0\right): c_{0} t=2|\gamma(s)-\mathbf{x}|\right\},
$$

which consists of circles centered at $\gamma(s)=\left(\gamma_{1}(s), \gamma_{2}(s), \gamma_{3}(s)\right)$ with radius $\sqrt{c_{0}^{2} t^{2} / 4-\gamma_{3}^{2}(s)}$.

ii.) Bi-static SAR: We begin by assuming that the transmitting antenna and receiving antenna are located on separate airborne platforms, and hence move along different paths $\gamma_{T}(s) \in \mathbb{R}^{3}$ and $\gamma_{R}(s) \in \mathbb{R}^{3}$, respectively. Then the data can be written ${ }^{2}$

$$
d(s, t)=\mathcal{F}[T](s, t)=\int e^{-i \omega\left(t-r_{s, \mathbf{x}}\right)} A(\boldsymbol{x}, s, \omega) T(\boldsymbol{x}) d \boldsymbol{x} d \omega,
$$

where

$$
r_{s, \mathbf{x}}=\left|\gamma_{T}(s)-\mathbf{x}\right| / c_{0}+\left|\gamma_{R}(s)-\mathbf{x}\right| / c_{0} .
$$

The method of stationary phase can be used to show that the main contributions to (4) come from the critical set

$$
X=\left\{\left(x_{1}, x_{2}, 0\right): c_{0} t=\left|\gamma_{T}(s)-\mathbf{x}\right|+\left|\gamma_{R}(s)-\mathbf{x}\right|\right\} .
$$

We conclude that our received data are closely related to integrals of $T$ along ellipses.

iii.) Hitchhiker SAR: This is a passive SAR modality which relies on sources of opportunity to image the ground irradiance. The system consists of airborne receiver(s) that traverse arbitrary paths over the scene of interest. The first step in received signal processing involves correlating the fast-time signal at different slow-time points. The tomographic reconstruction uses the correlated signal $d_{i j}$ defined by

$$
d_{i j}\left(s, s^{\prime}, t\right)=\int d_{i}(s, \tau) d_{j}\left(\tau-t, s+s^{\prime}\right) d \tau
$$


where

$$
d_{i}(s, t)=\int d_{i, \mathbf{y}}(s, t) d \mathbf{y}
$$

is the received signal at the $i^{\text {th }}$ receiver, which is a superposition of signals over all transmitters at locations $\mathbf{y}$. If we assume there are $N \geq 1$ receiving antennas, each following a trajectory $\gamma_{R_{i}}(s) \in \mathbb{R}^{3}, i=1, \ldots, N$ we can write $^{3}$ the Born approximated model of the correlated signal for receivers $i$ and $j$,

$$
d_{i j}\left(s, s^{\prime}, t\right)=\mathcal{F}[T]\left(s, s^{\prime}, t\right)=\int e^{-2 i \pi \omega\left(t-r_{i j}\left(s, s^{\prime}, \mathbf{x}\right)\right)} T(\boldsymbol{x}) A\left(\boldsymbol{x}, s, s^{\prime}, \omega\right) d \boldsymbol{x} d \omega, i, j=1, \ldots, N
$$

where

$$
r_{i j}(s, \mathbf{x})=\left|\mathbf{x}-\gamma_{R_{i}}(s)\right| / c_{0}-\left|\mathbf{x}-\gamma_{R_{j}}\left(s+s^{\prime}\right)\right| / c_{0}
$$

is the hitchhiker range and $A\left(\boldsymbol{x}, s, s^{\prime}, \omega\right)$ is again an amplitude which includes geometrical spreading factors, antenna beam patterns, and transmitter waveforms. Here $T$ is the scene irradiance. The leading-order contribution comes from

$$
X=\left\{\left(x_{1}, x_{2}, 0\right): c_{0} t=\left|\mathbf{x}-\gamma_{R_{i}}(s)\right|-\left|\mathbf{x}-\gamma_{R_{j}}\left(s+s^{\prime}\right)\right|\right\},
$$

which is a set of hyperbolas.

In general the data in all three modalities can be written as

$$
d(s, t)=\mathcal{F}[T](s, t)=\int e^{-i \omega(t-\phi(s, \mathbf{x}))} A(\boldsymbol{x}, s, \omega) T(\boldsymbol{x}) d \boldsymbol{x} d \omega .
$$

The general phase $\phi$ takes on forms such as

$$
\begin{aligned}
& \left.\phi(s, \mathbf{x})=r_{s, \mathbf{x}}=2|\gamma(s)-\mathbf{x}| / c_{0}\right) \\
& \phi(s, \mathbf{x})=r_{s, \mathbf{x}}=\left|\gamma_{T}(s)-\mathbf{x}\right| / c_{0}+\left|\gamma_{R}(s)-\mathbf{x}\right| / c_{0} \\
& \phi(s, \mathbf{x})=r_{i j}\left(s, s^{\prime}, \mathbf{x}\right)=\left|\mathbf{x}-\gamma_{R_{i}}(s)\right|-\left|\mathbf{x}-\gamma_{R_{j}}\left(s+s^{\prime}\right)\right|
\end{aligned}
$$

in the mono-SAR, bi-SAR, and hitchhiker SAR cases respectively.

Under mild conditions, the operator $\mathcal{F}$ of (12) that connects the scene $T$ to the data $d$ is a Fourier Integral Operator (FIO). A detailed definition of an FIO can be found in Appendix A. It is known that the behavior of an FIO is determined mainly by the critical set $X$ of the phase. In the case of mono-SAR, $X$ is the set of circles, for bi-static SAR, it is the set of ellipses, and in hitchhiker SAR it is the set of hyperbolas. An approximate inverse of $\mathcal{F}$ can be computed by another FIO which is described in the next section.

\subsection{Image Formation, Backprojection, and Microlocal Analysis}

Let us now focus primarily, for the sake of simplicity, on the general model for the data (12). To form an image we aim to invert (12) by applying an imaging operator $\mathcal{K}$ to the collected data. Because $\mathcal{F}$ is a Fourier integral operator we can compute an approximate inverse by means of another FIO. This FIO typically has the form of a filtered-backprojection (FBP) operator. In our case, we form an approximate inverse of $\mathcal{F}$ as a FBP operator, which first filters the data and then backprojects to obtain the image. Our imaging operator therefore takes on the form

$$
I(\boldsymbol{z})=\mathcal{K}[d](\boldsymbol{z}):=\int e^{i \omega(t-\phi(s, \mathbf{z}))} Q(\boldsymbol{z}, s, \omega) d \omega d(s, t) d s d t=\int e^{-i \omega \phi(s, \mathbf{z})} Q(\boldsymbol{z}, s, \omega) D(s, \omega) d \omega d s,
$$

where $\boldsymbol{z}=\left(z_{1}, z_{2}\right), \mathbf{z}=(\boldsymbol{z}, 0), D(s, \omega)$ is the Fourier transform of the data in fast time, and $Q$ is a filter which is determined in several different ways depending on the application. If we insert the equation for the data into above we have

$$
I(\boldsymbol{z})=\mathcal{K} \mathcal{F}[T](\boldsymbol{z})=\int e^{i \omega(\phi(s, \mathbf{x})-\phi(s, \mathbf{z}))} Q(\boldsymbol{z}, s, \omega) A(\boldsymbol{x}, s, \omega) d \omega d s T(\boldsymbol{x}) d \boldsymbol{x} .
$$


In synthetic aperture imaging we are especially interested in identifying singularities, or edges and boundaries of objects, from the scene of interest in our image. The study of singularities is part of microlocal analysis, which also encompasses FIO theory. This theory tells us the image-fidelity operator $\mathcal{L}=\mathcal{K} \mathcal{F}$ is a pseudodifferential operator, a special case of an FIO. This observation allows us to invoke the pseudolocal property which states that the visible singularities in the scene $T$ are preserved in our image, in the sense that edges appear with the correct location and orientation. For more details on the theory of microlocal analysis and pseudodifferential operators, see Appendix B.

\section{GENERALIZED LIKELIHOOD RATIO TEST}

The generalized likelihood ratio test (GLRT) is used in detection and estimation theory to address the problem of detecting a target or signal that depends on unknown parameters. One calculates a test statistic via the likelihood ratio to determine whether or not the target is present, and performs a maximum-likelihood-estimation step to determine unknown parameters. In our application of synthetic aperture imaging we seek to determine whether a target or object of interest is present in our data and to identify important unknowns such as the object's location or edges.

For simplicity, we assume that the object in our scene is a point scatterer with scattering strength $C$ : $T(\boldsymbol{x})=C \delta(\boldsymbol{x}-\boldsymbol{y})$, where $\boldsymbol{y}$ is the unknown location of the object in our scene. In this case, (12) has the form

$$
d_{\boldsymbol{y}}(s, t)=\mathcal{F}[T](s, t)=C \int e^{-i \omega(t-\phi(s, \mathbf{y}))} A(\boldsymbol{y}, s, \omega) d \omega .
$$

We note that $A$ depends on $\boldsymbol{y}$ only through the geometrical spreading factors, which are slowly varying. Consequently we neglect the dependence on $\boldsymbol{y}$ and write

$$
A(\boldsymbol{y}, s, \omega) \approx \tilde{A}(s, \omega) .
$$

Thus we write

$$
\tilde{d}_{\boldsymbol{y}}(s, t)=\mathcal{F}[T](s, t)=C \int e^{-i \omega(t-\phi(s, \mathbf{y}))} \tilde{A}(s, \omega) d \omega .
$$

In order to determine whether the target is present, and if it is present determine its location, we consider the hypothesis testing problem

$$
\begin{aligned}
& H_{0}: \quad m(s, t)=n(s, t) \\
& H_{\boldsymbol{y}}: \quad m(s, t)=\tilde{d}_{\boldsymbol{y}}(s, t)+n(s, t)
\end{aligned}
$$

where $m(s, t)$ is our measured data and $n(s, t)$ is additive white Gaussian noise.

Also we assume that our additive white Gaussian noise is stationary with the covariance kernel

$$
K\left(s_{1}, s_{2}, t_{1}, t_{2}\right)=E\left[n\left(s_{1}, t_{1}\right) \overline{n\left(s_{2}, t_{2}\right)}\right]=\int \sigma^{2} e^{i \omega\left(t_{1}-t_{2}\right)} d \omega e^{i \alpha\left(s_{1}-s_{2}\right)} d \alpha
$$

for $\left(s_{1}, t_{1}\right)$ and $\left(s_{2}, t_{2}\right) \in S$, where $S=\left\{(s, t): s_{0} \leq s \leq s_{N}, t_{0} \leq t \leq t_{N}\right\}$ is the index set on which our stochastic processes $m$ and $n$ are defined. In addition note that $\sigma$ is a constant.

In this particular problem because our null hypothesis data does not depend on our unknown parameter we can perform both steps of the GLRT simultaneously. If our data were discrete we would simply calculate the likelihood ratio for our test statistic by finding the ratio of the probability density functions of the data under each hypothesis. We calculate this test statistic $\lambda(\boldsymbol{y})$ for each possible $\boldsymbol{y}$ in the parameter space $\mathbf{X}$, and then to obtain an estimate $\widetilde{\boldsymbol{y}}$ of the unknown $\boldsymbol{y}$ we maximize over all $\boldsymbol{y} \in \mathbf{X}$, i.e.

$$
\begin{aligned}
\lambda(\boldsymbol{y}) & =\frac{f_{H_{\boldsymbol{y}}}(m)}{f_{H_{0}}(m)} \\
\widetilde{\boldsymbol{y}} & =\underset{\boldsymbol{y} \in \mathbf{X}}{\operatorname{argmax}} \lambda(\boldsymbol{y}) .
\end{aligned}
$$


In our case the data or measurements are continuous rather than discrete, which requires us to use a different method for calculating the likelihood ratio. This issue arises because one cannot write the probability density function of a continuous random process explicity. We instead look to the Radon-Nikodym derivative of a measure with respect to another measure (see Appendix C) to find our explicit formula for the likelihood ratio or test statistic. We let $P_{H_{0}}$ and $P_{H_{y}}$ be the probability measures describing the data under the null hypothesis, $H_{0}$, and the alternative hypothesis, $H_{\boldsymbol{y}}$, respectively. Consequently the Radon-Nikodym derivative of $P_{H_{\boldsymbol{y}}}$ with respect to $P_{H_{0}}$ can be written

$$
p=\frac{d P_{H_{y}}}{d P_{H_{0}}} .
$$

A sufficient and necessary condition for the existence of $p$ is that $P_{H_{y}}$ be absolutely continuous with respect to $P_{H_{0}}$, i.e. for every measurable subset $B$ of the sample space $\Omega$ such that $P_{H_{0}}[B]=0$, we have $P_{H_{y}}[B]=0$ as well. It turns out that we can say with certainty that this condition holds if $\tilde{d}_{\boldsymbol{y}}(s, t) \in H(K)$, that is, if our signal is a member of the reproducing kernel Hilbert space generated by the covariance kernel of the noise $K$. If this condition is satisfied then we can write down an explicit expression for the Radon-Nikodym derivative, or likelihood ratio, in terms of inner products on the reproducing kernel Hilbert space $H(K)$. This explicit expression is

$$
p[m(s, t)]=\exp \left\{\left(m, \tilde{d}_{\boldsymbol{y}}\right)_{K}-\frac{1}{2}\left(\tilde{d}_{\boldsymbol{y}}, \tilde{d}_{\boldsymbol{y}}\right)_{K}\right\},
$$

where $(\cdot, \cdot)_{K}$ denotes the inner product on $H(K)$. Note $\left(m, \tilde{d}_{\boldsymbol{y}}\right)_{K}$ is not a true inner product because $m(s, t)$ is not a member of $H(K)$. We need to modify the expression for the inner product to obtain this term in the likelihood ratio. For more details on the Radon-Nikodym derivative and reproducing kernel Hilbert spaces, see Appendix C.

The reproducing kernel Hilbert space $H(K)$ consists of functions $g$ defined on $S$ of the form

$$
g(s, t)=\int G(s, \omega) e^{i t \omega} d \omega
$$

where $G(s, \omega)=\sigma^{2} g^{*}(s, \omega)$ (for some unique function $g^{*}(s, \omega)$ ) is the Fourier transform of $g(s, t)$. The norm of $g$ satisfies

$$
\|g\|_{K}^{2}=\frac{1}{\sigma^{2}} \int|G(s, \omega)|^{2} d s d \omega<\infty .
$$

The integral representation theorem (see appendix C for more details) implies that the "inner product" with $m$ takes the form

$$
(m, g)_{K}=\frac{1}{\sigma^{2}} \int \overline{G(s, \omega)} M(s, \omega) d s d \omega
$$

where $M(s, \omega)$ is the Fourier transform of $m$.

We write (18) in the form

$$
\tilde{d}_{\boldsymbol{y}}(s, t)=\int e^{-i \omega t} \tilde{D}_{\boldsymbol{y}}(s, \omega) d \omega
$$

where

$$
\tilde{D}_{\boldsymbol{y}}(s, \omega)=C e^{i \omega \phi(s, \boldsymbol{y})} \tilde{A}(s, \omega) .
$$

Therefore we see that $\tilde{d}_{\boldsymbol{y}}$ can be expressed in the form of equation (24) and consequently $\tilde{d}_{\boldsymbol{y}} \in H(K)$. We conclude that the first term in the likelihood ratio (23) may be written as

$$
\left(m, \tilde{d}_{\boldsymbol{y}}\right)_{K}=\frac{C}{\sigma^{2}} \int e^{-i \omega \phi(s, \boldsymbol{y})} \overline{\tilde{A}(s, \omega)} M(s, \omega) d s d \omega .
$$

Similarly, we compute

$$
\left(\tilde{d}_{\boldsymbol{y}}, \tilde{d}_{\boldsymbol{y}}\right)_{K}=\frac{1}{\sigma^{2}} \int|\tilde{A}(s, \omega) C|^{2} d s d \omega
$$


We thus have the expression for the log of the likelihood ratio $p$, which we take as our test statistic $\lambda(\boldsymbol{y})$,

$$
\log [p(m)]=\lambda(\boldsymbol{y})=\frac{C}{\sigma^{2}} \int e^{-i \omega \phi(s, \boldsymbol{y})} \tilde{\tilde{A}(s, \omega)} M(s, \omega) d s d \omega-\frac{1}{2 \sigma^{2}} \int|\tilde{A}(s, \omega) C|^{2} d s d \omega .
$$

Note that the second term of (31) does not depend on the unknown parameter $\boldsymbol{y}$ and therefore does not provide any information for our estimation and detection task. We can therefore neglect the second term of (31) and obtain the following expression for the test statistic at each possible location $\boldsymbol{y}$ :

$$
\lambda(\boldsymbol{y})=\int e^{-i \omega \phi(s, \boldsymbol{y})} \overline{\tilde{A}(s, \omega)} M(s, \omega) d s d \omega .
$$

If we take the inverse Fourier transform of $M(s, \omega)$ we then obtain the time domain version of the test statistic,

$$
\lambda(\boldsymbol{y})=\int e^{i \omega(t-\phi(s, \boldsymbol{y}))} \overline{\tilde{A}(s, \omega)} d \omega m(s, t) d s d t .
$$

We see that (33) is a special case of (14), where in (33) we have used $m$ rather than $d$ to denote the collected data. In (33), the filter $Q$ of (14) is the matched filter, namely the complex conjugate of the amplitude $\tilde{A}$.

We have shown that, with this choice of filter, the FBP image is equivalent to the test statistic calculated at each possible location $\boldsymbol{y}$. We observe that one may think of the values of the test statistic for each $\boldsymbol{y}$ as a value assigned to a pixel. All these pixel values can be plotted to obtain a corresponding 'test statistic image', which as we have shown, is equivalent to a filtered-backprojection image formed with a matched filter.

The final step of the detection and estimation problem is to estimate the unknown $\boldsymbol{y}$. We achieve this simply by maximizing the above expression over all possible $\boldsymbol{y} \in \mathbf{X}$, i.e.

$$
\widetilde{\boldsymbol{y}}=\underset{\boldsymbol{y} \in \mathbf{X}}{\operatorname{argmax}} \lambda(\boldsymbol{y})=\underset{\boldsymbol{y} \in \mathbf{X}}{\operatorname{argmax}} \int e^{i \omega(t-\phi(s, \boldsymbol{y}))} \overline{\tilde{A}(s, \omega)} d \omega m(s, t) d s d t .
$$

This result can easily be extended to the case when our additive noise is colored by replacing $\sigma^{2}$ with a spectral density function $f(\omega)$. In this case, we obtain a similar result to above, but instead the filter is a matched filter in conjuction with a whitening filter, as one would expect.

\section{CONCLUSION}

In this paper we have put three different modalities of SAR into a common framework and then introduced two different image formation techniques. We have demonstrated that these techniques, which arise from different theories, lead to equivalent images under the assumption of stationary white Gaussian noise. That is, the test statistic image obtained from the GLRT technique is of the form of a FBP image, in which the filter chosen is a matched filter. 


\section{APPENDIX A. FOURIER INTEGRAL OPERATORS}

Definition. A Fourier integral operator $(\mathrm{FIO}) \mathcal{P}$ of order $m$ is defined as

$$
\mathcal{P} u=\int e^{i \Phi(\boldsymbol{x}, \boldsymbol{y}, \boldsymbol{\xi})} p(\boldsymbol{x}, \boldsymbol{y}, \boldsymbol{\xi}) u(\boldsymbol{y}) d \boldsymbol{y} d \boldsymbol{\xi},
$$

where $p(\boldsymbol{x}, \boldsymbol{y}, \boldsymbol{\xi}) \in C^{\infty}\left(X \times Y \times \mathbb{R}^{n}\right)$ satisfies the estimate: for every compact set $K \subset X \times Y$ and for every multi-index $\alpha, \beta, \gamma$, there is a constant $C=C(K, \alpha, \beta, \gamma)$ such that

$$
\left|\partial_{\boldsymbol{\xi}}^{\alpha} \partial_{\boldsymbol{x}}^{\beta} \partial_{\boldsymbol{y}}^{\gamma} p(\boldsymbol{x}, \boldsymbol{y}, \boldsymbol{\xi})\right| \leq C(1+|\boldsymbol{\xi}|)^{m-|\alpha|}
$$

for all $\boldsymbol{x}, \boldsymbol{y} \in K$ and for all $\boldsymbol{\xi} \in \mathbb{R}^{n}$. Also $\Phi$ must be a phase function, i.e. if,

1.) $\Phi$ is positively homogeneous of degree 1 in $\boldsymbol{\xi}$. That is $\Phi(\boldsymbol{x}, \boldsymbol{y}, r \boldsymbol{\xi})=r \Phi(\boldsymbol{x}, \boldsymbol{y}, \boldsymbol{\xi})$ for all $r>0$.

2.) $\left(\partial_{\boldsymbol{x}} \Phi, \partial_{\boldsymbol{\xi}} \Phi\right)$ and $\left(\partial_{\boldsymbol{y}} \Phi, \partial_{\boldsymbol{\xi}} \Phi\right)$ do not vanish for all $(\boldsymbol{x}, \boldsymbol{y}, \boldsymbol{\xi}) \in X \times Y \times \mathbb{R}^{n} \backslash\{0\}$.

The phase variable $\boldsymbol{\xi}$ is the analogue of $\omega$ in (12). One example of an FIO is a pseudodifferential operator, which is an FIO with a phase function of the form $\Phi(\mathbf{x}, \mathbf{z}, \boldsymbol{\xi})=(\mathbf{z}-\mathbf{x}) \cdot \boldsymbol{\xi}$.

\section{APPENDIX B. MICROLOCAL ANALYSIS}

The mathematical theory of microlocal analysis is a way of analyzing singularities, in our case these singularities are the edges and boundaries we seek to identify. We begin our discussion of the theory by describing the way singularities are characterized in microlocal analysis, by their location and direction. We define the singular structure of a function by its wavefront set which is the collection of singular points and their associated directions. Intuitively the directions included in the wavefront set are those in which the function oscillates most in the frequency domain. A formal definition of the wavefront set is as follows,

Definition. The point $\left(\boldsymbol{y}, \boldsymbol{\xi}_{0}\right)$ is not in the wavefront set $W F(f)$ of the function $f$ if there is a smooth cutoff function $\psi$ with $\psi(\boldsymbol{y}) \neq 0$, for which the Fourier transform $\widehat{(f \psi)}(\lambda \boldsymbol{\xi})$ decays rapidly (i.e. faster than any polynomial in $1 / \lambda)$ as $\lambda \rightarrow \infty$, uniformly for $\boldsymbol{\xi}$ in a neighborhood of $\boldsymbol{\xi}_{0}$.

We can break down this definition into three steps to determine if a point is in the wavefront set: i.) localize around $\boldsymbol{y}$ by multiplying by a smooth cutoff function $\psi$ supported in the neighborhood of $\boldsymbol{y}$, ii.) Fourier transform $f \psi$, and iii.) study the decay of the Fourier transform in the direction $\boldsymbol{\xi}_{0}$. If the Fourier transform decays rapidly in that direction the point is not in the wavefront set. We include two examples to make this concept more concrete.

Example 1 A point scatterer, i.e. if $f(\boldsymbol{x})=\delta(\boldsymbol{x})$, then

$$
W F(f)=\{(0, \boldsymbol{\xi}): \boldsymbol{\xi} \neq 0\}
$$

Example 2 A line, i.e. if $f(\boldsymbol{x})=\delta(\boldsymbol{x} \cdot \boldsymbol{\nu})$, then

$$
W F(f)=\{(\boldsymbol{x}, \alpha \boldsymbol{\nu}): \boldsymbol{x} \cdot \boldsymbol{\nu}=0, \alpha \neq 0\}
$$

We use the concepts of microlocal analysis to analyze how singularities in the scene or in $T$ correspond to singularities in the image. Recall the equation for the image from (15), which we can rewrite as,

$$
I(\boldsymbol{z})=\mathcal{L}[T](\boldsymbol{z})=\int L(\boldsymbol{z}, \boldsymbol{x}) T(\boldsymbol{x}) d \boldsymbol{x},
$$


where $\mathcal{L}=\mathcal{K} \mathcal{F}$ is known as the image-fidelity operator and $L$, the kernel of $\mathcal{L}$, is called the point-spread function (PSF). We will show that $\mathcal{L}$ is a pseudodifferential operator which, recall from Appendix A, has a kernel of the form,

$$
L(\boldsymbol{z}, \boldsymbol{x})=\int e^{i(\mathbf{x}-\mathbf{z}) \cdot \boldsymbol{\xi}} p(\boldsymbol{z}, \boldsymbol{x}, \boldsymbol{\xi}) d \boldsymbol{\xi},
$$

where $p$ must satisfy certain symbol estimates as in the defintion for a general FIO. It is essential that our imagefidelity operator be a pseudodifferential operator because this class of FIOs has the property that they map wavefront sets in a desirable way. This property is known as the pseudolocal property which states $W F(\mathcal{L} f) \subseteq W F(f)$, that is, the operator $\mathcal{L}$ does not increase the wavefront set. In the imaging setting this says that the visible singularities of the scene, $T$, are put in the correct location with the correct orientation. This is desirable for our application because we can say with certainty that no singularities or edges in the image are the result of artifacts. However, we note that it is possible some edges present in $T$ may not appear in the image, especially if the viewing aperture is limited. We now show that the filtered-backprojection operator is indeed a pseudodifferential operator and therefore produces an image where edges are in the correct location and have the correct orientation.

In order to demonstrate that $L$ is the kernel of a pseudodifferential operator our goal is to determine $\mathcal{K}$, the imaging operator, so that $L$ is of the form in equation (36). First we must ensure $\mathcal{K}$ is an FIO by requiring $Q$ to satisfy a symbol estimate similar to that of $p$, i.e. we assume for some $m_{Q}$,

$$
\sup _{(s, \boldsymbol{z}) \in K}\left|\partial_{\omega}^{\alpha} \partial_{s}^{\beta} \partial_{z_{1}}^{\rho_{1}} \partial_{z_{2}}^{\rho_{2}} Q(\boldsymbol{z}, s, \omega)\right| \leq C_{0}\left(1+\omega^{2}\right)^{\left(m_{Q}-|\alpha|\right) / 2}
$$

where $K$ is any compact subset of $\mathbb{R} \times \mathbb{R}^{2}$ and $C_{0}=C_{0}\left(K, \alpha, \beta, \rho_{1}, \rho_{2}\right)$.

We now must show that the phase of $\mathcal{L}$ can be written in the form $\Phi(\mathbf{x}, \mathbf{z}, \boldsymbol{\xi})=i(\mathbf{x}-\mathbf{z}) \cdot \boldsymbol{\xi}$. Recall that our image is given by

$$
I(\boldsymbol{z})=\mathcal{K} \mathcal{F}[T](\boldsymbol{z})=\mathcal{L}[T](\boldsymbol{z})=\int e^{i \omega(\phi(s, \mathbf{x})-\phi(s, \mathbf{z}))} Q(\boldsymbol{z}, s, \omega) A(\boldsymbol{x}, s, \omega) d \omega d s T(\boldsymbol{x}) d \boldsymbol{x} .
$$

The point-spread function $L$ is

$$
L(\boldsymbol{z}, \boldsymbol{x})=\int e^{i \omega(\phi(s, \mathbf{x})-\phi(s, \mathbf{z}))} Q(\boldsymbol{z}, s, \omega) A(\boldsymbol{x}, s, \omega) d \omega d s .
$$

In order to determine how close the phase is to that of a pseudodifferential operator, one applies the method of stationary phase to the $s$ and $\omega$ integrals. The stationary phase method gives us an approximate formula for the large-parameter behavior of our oscillatory integral. First we introduce a large parameter $\beta$ by the change of variables $\omega=\beta \omega^{\prime}$. The stationary phase theorem tells us that the main contribution to the integral comes from the critical points of the phase, i.e. points satisfying the critical conditions

$$
\begin{aligned}
& 0=\nabla_{\omega^{\prime}} \Phi \propto \phi(s, \mathbf{x})-\phi(s, \mathbf{z}) \\
& 0=\nabla_{s} \Phi \propto \nabla_{s}(\phi(s, \mathbf{x})-\phi(s, \mathbf{z})) .
\end{aligned}
$$

One of the solutions of the above equations is the critical point $\mathbf{x}=\mathbf{z}$. Other critical points lead to artifacts in the image, however their presence depends on the measurement geometry and the antenna beam pattern. We assume the flight trajectories and antenna beam patterns are such that we obtain only the critical point when $\mathbf{x}=\mathbf{z}$. In the neighborhood of the point $\mathbf{x}=\mathbf{z}$, we use a Taylor expansion of the exponent to force the phase to look like that of a pseudodifferential operator. We make use of the formula

$$
f(\mathbf{x})-f(\mathbf{z})=\int_{0}^{1} \frac{d}{d \boldsymbol{\mu}} f(\mathbf{z}+\boldsymbol{\mu}(\mathbf{x}-\mathbf{z})) d \boldsymbol{\mu}=\left.(\mathbf{x}-\mathbf{z}) \cdot \int_{0}^{1} \nabla f\right|_{\mathbf{z}+\boldsymbol{\mu}(\mathbf{x}-\mathbf{z})} d \boldsymbol{\mu}
$$

where in our case $f(\mathbf{z})=\omega \phi(s, \mathbf{z})$. We then make the Stolt change of variables

$$
(s, \omega) \rightarrow \boldsymbol{\xi}=\mathbf{\Xi}(s, \omega, \mathbf{x}, \mathbf{z})=\left.\int_{0}^{1} \nabla f\right|_{\mathbf{z}+\boldsymbol{\mu}(\mathbf{x}-\mathbf{z})} d \boldsymbol{\mu} .
$$


This change of variables allows us to obtain a new form of the point-spread function,

$$
L(\boldsymbol{z}, \boldsymbol{x})=\int e^{i(\mathbf{x}-\mathbf{z}) \cdot \xi} Q A(\boldsymbol{x}, s(\boldsymbol{\xi}), \omega(\boldsymbol{\xi}))\left|\frac{\partial(s, \omega)}{\partial \boldsymbol{\xi}}\right| d \boldsymbol{\xi} .
$$

From this expression, we see the phase of $\mathcal{L}$ is of the form required of pseudodifferential operators. With the symbol estimate requirements made on $Q$, we have shown that our image-fidelity operator is indeed a pseudodifferential operator. We conclude that the pseudolocal property holds and therefore our microlocal-analysis-based reconstruction method preserves the singularities or edges in our scene of interest.

\section{APPENDIX C. THE RADON-NIKODYM DERIVATIVE AND REPRODUCING KERNEL HILBERT SPACES}

The Radon-Nikodym derivative is the result of the Radon-Nikodym theorem ${ }^{5}$ which states,

Theorem. Suppose that $P_{H_{1}}$ and $P_{H_{0}}$ are $\sigma$-finite measures on $(\Omega, \mathcal{B})$ and that $P_{H_{1}}$ is absolutely continuous with respect to $P_{H_{0}}$. Then there exists a measurable function $p: m \rightarrow \mathbb{R}$ such that

$$
P_{H_{1}}[B]=\int_{B} p d P_{H_{0}}, \forall B \in \mathcal{B}
$$

Moreover $p$ is uniquely defined except possibly on a set $B_{0}$ with $P_{H_{0}}\left(B_{0}\right)=0$. The function $p$ is called the Radon-Nikodym derivative of $P_{H_{1}}$ with respect to $P_{H_{0}}$ and is written

$$
p=\frac{d P_{H_{1}}}{d P_{H_{0}}} .
$$

As stated in section 3 , this function $p$ exists for the measures associated with the data under each hypothesis if $d \in H(K)$ where $H(K)$ is the reproducing kernel Hilbert space generated by $K$, the covariance kernel of the noise. It is a known result ${ }^{6}$ that every covariance kernel generates a unique reproducing kernel Hilbert space. A formal definition is as follows,

Definition. ${ }^{6}$ A Hilbert space $H(K)$ is said to be a reproducing kernel Hilbert space, with reproducing kernel $K$, if the members of $H(K)$ are functions on some set $S$, and if there exists a kernel on $S \times S$ having 2 properties: for every $s \in S$ where $K(\cdot, s)$ is a function defined on $S$, with value at $t \in S$ equal to $K(t, s)$,

$$
\begin{gathered}
K(\cdot, s) \in H(K) \\
(g, K(\cdot, s))_{K}=g(s), \forall g \in H(K)
\end{gathered}
$$

where $(\cdot, \cdot)_{K}$ denotes the inner product on $H(K)$.

Again as stated in the GLRT section, if $d \in H(K)$ then $p$ exists and is defined by equation (23). This formula is a result of the theorem ${ }^{6}$

Theorem. Let $P_{H_{1}}$ be the probability measure induced on the space of sample functions of a time series $\{m(s, t),(s, t) \in S\}$ with covariance kernel $K$ and mean value function $d$. Assume that either (i) $\mathrm{S}$ is countable or (ii) $\mathrm{S}$ is a separable metric space, $K$ is continuous, and the stochastic process $\{m(s, t),(s, t) \in S\}$ is separable. Let $P_{H_{0}}$ be the probability measure corresponding to the Gaussian process with covariance kernel $K$ and with zero mean. Then $P_{H_{1}}$ and $P_{H_{0}}$ are absolutely continuous with respect to one another, or orthogonal, depending on whether $d$ does belong or does not belong to $H(K)$. If $d \in H(K)$ then the Radon-Nikodym derivative of $P_{H_{1}}$ with respect to $P_{H_{0}}$ is given by

$$
p[m(s, t)]=\exp \left\{(m, d)_{K}-\frac{1}{2}(d, d)_{K}\right\} .
$$


We can also describe the specific reproducing kernel Hilbert space, $H(K)$, which is generated by the covariance kernel of a stationary process. The structure of the space is given by the integral representation theorem, ${ }^{6}$ which we state in terms of $K$ in the stationary case. The theorem follows below.

Integral Representation Theorem for Stationary Processes. Let $S=[s: \infty<s<\infty]$ and let $[m(s), s \in S]$ be a stationary time series with spectral density function $f(\omega)$ so that

$$
K(s, t)=\int e^{i \omega(s-t)} f(\omega) d \omega
$$

Then $H(K)$ consists of all functions $g$ on $S$ of the form

$$
g(s)=\int G(\omega) e^{i \omega s} d \omega
$$

where $G(\omega)=g^{*}(\omega) f(\omega)$ (for some unique function $g^{*}(\omega)$ ), for which the norm

$$
\|g\|_{K}^{2}=\int \frac{|G(\omega)|^{2}}{f(\omega)} d \omega
$$

is finite. The corresponding random variable $(m, g)_{K}$ can be expressed in terms of the spectral representation of $m$. If

$$
m(s)=\int e^{i \omega s} d Z(\omega)
$$

then

$$
(m, g)_{K}=\int \frac{\overline{G(\omega)}}{f(\omega)} d Z(\omega)
$$

\section{ACKNOWLEDGMENTS}

This work was supported by by the Air Force Office of Scientific Research under agreements number FA955009-1-0013 and FA9550-07-1-0363, and by the Air Force Research Laboratory* under agreement number FA865008-C-1322.

\section{REFERENCES}

1. C.J. Nolan and M. Cheney, "Synthetic aperture inversion", Inverse Problems, vol. 18, pp. 221-236, 2002.

2. C.E. Yarman, B. Yazici, and M. Cheney, "Bistatic synthetic aperture radar imaging with arbitrary trajectories", IEEE Transactions in Image Processing, Vol. 17, No: 1, pp: 84-93, 2008.

3. C.E. Yarman, B. Yazici "Synthetic aperture hitchhiker imaging", IEEE Transactions in Image Processing, Vol. 17, No. 11, pp: 2156-2173, 2008.

4. Rossi, D.J. and Willsky, A.S., "Reconstruction from Projections Based on Detection and Estimation of Objects - Part I: Performance Analysis and Part II: Robustness Analysis", IEEE Trans. on Acoustics, Speech, and Signal Processing, Vol. 32, No. 4, pp. 886-906, Aug. 1984.

5. Royden, H.L., Real Analysis, Macmillan, New York, 1968.

6. Parzen, E., Time Series Analysis Papers, Holden-Day Series in Time Series Analysis, 1967.

\footnotetext{
${ }^{*}$ Consequently the U.S. Government is authorized to reproduce and distribute reprints for Governmental purposes notwithstanding any copyright notation thereon. The views and conclusions contained herein are those of the authors and should not be interpreted as necessarily representing the official policies or endorsements, either expressed or implied, of the Air Force Research Laboratory or the U.S. Government.
} 